\title{
Liposomal cytarabine in central nervous system disease of haematological malignancies: more effective but more toxic?
}

\author{
Mar Tormo Díaz
}

$I^{\mathrm{n}}$

nfiltration of the central nervous system (CNS) is a serious complication in patients with haematological malignancies and is associated with a poor prognosis. CNS involvement in patients with acute lymphoblastic leukaemia (ALL) at the time of presentation is uncommon and has been reported in 5-7\% of patients [1]. Without prophylaxis, CNS recurrence occurs in approximately $30 \%$ of adult patients and up to $75 \%$ of children with ALL in complete response [2, 3]. However, with therapies that incorporates CNS prophylaxis, 5-year event-free survival rates of approximately $80 \%$ and $50 \%$ have been achieved in paediatric and adult ALL, respectively [4]. In patients with lymphoma, histologic subtype is one of the main risk factors for CNS recurrence. There is consensus on the need for CNSdirected therapy in aggressive lymphomas such as Burkitt's lymphoma and lymphoblastic lymphoma, which have a risk of recurrence, in the absence of prophylaxis, of up to 20$25 \%$. However, there is controversy regarding this therapy for other aggressive lymphomas, such as mantle cell lymphoma, anaplastic lymphoma, peripheral $\mathrm{T}$ lymphomas and especially diffuse large B-cell lymphomas. Several studies have estimated the risk of CNS recurrence as about $5 \%$.

The treatment of disseminated lymphomatous meningitis requires long exposure of the malignant cells to a high concentration of antineoplastic agent to achieve a sufficiently cytostatic effect. CNS-directed therapy is based on the administration of high-dose systemic chemotherapy with drugs that can pass through the blood-brain barrier, such as methotrexate (MTX) and cytarabine, cranial or craniospinal irradiation, and intrathecal (IT) administration of MTX and/or cytarabine [5]. Systemic chemotherapy alone is not adequate for CNS prophylaxis and treatment, since it is difficult to maintain prolonged therapeutic concentrations of drugs in the cerebrospinal fluid (CSF).

M. Tormo Díaz (凶)

Hematology and Oncology Service

Clinic Universitary Hospital and INCLIVA Health Research Institute

Av. Blasco Ibáñez 17

ES-46010 Valencia, Spain

e-mail: tormo_mar@gva.es
Moreover, radiation can be effective but is often related to late adverse effects, like secondary neoplasms, neurocognitive dysfunction and neurotoxicity [6]. IT chemotherapy is a standard treatment for lymphomatous meningitis, but only few drugs can be used in this way. In general, treatments use IT MTX, cytarabine or thiotepa. The main problem with these drugs is their rapid clearance from the CSF, meaning 2-3 administrations per week are required, either by lumbar puncture or an implanted Omaya reservoir. This is inconvenient for patients, presents multiple opportunities for injection-related trauma or infection and is a very stressful process for patients and their carers.

Recently, a liposomal formulation of cytarabine (DepoCyte $^{\circledR}$, Mundipharma Pharmaceuticals SL, Cambridge, UK) has been developed that has a significantly longer CSF half-life. After administration of a 50-mg injection, free cytarabine is detectable for up to 14 days. The prolonged activity allows better distribution of the drug in the whole CSF surrounding the brain [7]. Liposomal cytarabine has allowed the frequency of administration to be reduced to once every 2 weeks during the induction and consolidation phases, and once every 4 weeks during the maintenance phase. All patients being treated with liposomal cytarabine should receive concomitant oral or IT dexamethasone for five days to reduce the risk of chemical arachnoiditis.

The efficacy and tolerability of IT liposomal cytarabine have been demonstrated in two randomised controlled trials developed in patients with neoplastic meningitis associated with lymphoma [8] and solid tumours [9], although a survival advantage was not demonstrated in the latter study. Based on its significant efficacy, the drug was approved by the Food and Drug Administration and EMEA for the treatment of adults with lymphomatous meningitis. There is less evidence on the use of liposomal cytarabine in children and the recommended dose in a phase I study in patients aged between 3 and 21 years with haematological malignancies or solid tumours was $35 \mathrm{mg}$ [10].

There are concerns about the toxicity associated with liposomal cytarabine, especially when they are used concomitantly with other drugs that cross the blood-brain barrier. The M.D. Anderson experience in adults diagnosed with ALL and treated with a hyper-CVAD regimen that 
included high-dose MTX and cytarabine, and the substitution of conventional prophylactic IT treatment with liposomal cytarabine, was associated with a high percentage of neurotoxicity (16\%) including encephalopathy, cauda equina syndrome, seizure and pseudotumour cerebri. This toxicity occurred despite having separated the administration of liposomal cytarabine from cytarabine administered systemically by 12 days [11]. They conclude that severe neurotoxicity may occur after concurrent administration of IT liposomal cytarabine and systemic chemotherapy agents that penetrate the blood-brain barrier. Radiation therapy may also potentiate the neurotoxicity associated with liposomal cytarabine. In the randomised trials from Glantz et al., most adverse events were transient and resolved by the end of the treatment cycle in which they occurred. The only adverse event that happened in more than $10 \%$ of treatment cycles was headache, which was more frequent in the liposomal cytarabine arm than in the free cytarabine arm (27\% vs. $2 \%$ of cycles). However, grade 3 headache occurred over only $5 \%$ of the liposomal cytarabine cycles. The other common side effect was arachnoiditis, which occurred in $22 \%$ of cases of patients treated with liposomal cytarabine vs. $13 \%$ of patients treated with free cytarabine. There were no significant differences in episodes of severe arachnoiditis between the two treatment arms. We must take into account in this study that concurrent systemic chemotherapy for treatment of disease outside of the meninges was permitted with the exception of high-dose MTX, cytarabine or thiote$\mathrm{pa}$, which may explain the favourable toxicity profile [8].
Recently, one study has reported the results of a prospective non-randomised multicentre phase II trial for the treatment of CNS relapse in adult patients with ALL and Burkitt's lymphomas with liposomal cytarabine [12]. This study involved a negative group of heavily pretreated patients with ALL and very aggressive lymphoma; the overall rate of CNS cytological response was $74 \%$. On the other hand, $89 \%$ patients experienced an adverse event of some degree, of which $68 \%$ were grade 3 or 4 and $32 \%$ related to liposomal cytarabine.

The article published in this issue by Navajas et al. shows the results of treatment with liposomal cytarabine in a group of paediatric patients from nine Spanish centres with primary CNS tumours and leptomeningeal involvement. Overall, the results were good, with a response rate of $58 \%$, and although $55 \%$ of patients experienced side effects, these were mild or moderate. None of these patients received high doses of drugs that cross the blood brainbarrier and only two received concurrent radiotherapy [13].

In conclusion, cytarabine liposome is effective in the treatment of meningitis lymphomatous of ALL, aggressive lymphomas and probably in brain tumours with leptomeningeal involvement. However, additional studies should be conducted in these patients to establish how to use this agent in combination with systemic chemotherapy, especially with drugs that penetrate the blood-brain barrier. Prospective trials are necessary to define the optimal schedule, frequency and combination with systemic therapy.

\section{References}

1. Lazarus HM, Richards SM, Chopra R et al (2006) Medical Research Council (MRC)/National Cancer Research Institute (NCRI) Adult Leukemia Working Party of the United Kingdom and the Eastern Cooperative Oncology Group. Central nervous system involvement in adult acute lymphoblastic leukemia at diagnosis: result from the international ALL trial MRC UKALL-XII/ECOG E2993. Blood 108:465-472

2. Evans AE, Gilbert ES, Zandstra R (1970) The increasing incidence of central nervous system leukemia in children. Cancer 26:404-409

3. Omura GA, Moffitt S, Vogler WR, Salter MM (1980) Combination chemotherapy of adult acute lymphoblastic leukemia with randomized central nervous prophylaxis. Blood 55:199-204

4. Pui CH, Evans WE (2006) Treatment of acute lymphoblastic leukemia. N Engl J Med 354:166178
5. Pui CH, Thiel E (2009) Central nervous system disease in hematological malignancies: historical perspective and practical applications. Semin Oncol 36[4 Suppl 2]:S2-S16

6. Laack NN, Brown PD (2004) Cognitive sequelae of brain radiation in adults. Semin Oncol 31:702-713

7. Phuphanich S, Maria B, Braeckman R, Chamberlain M (2007) A pharmacokinetic study of intra-CSF administered encapsulated cytarabine (DepoCyt) for the treatment of neoplastic meningitis in patients with leukemia, lymphoma, or solid tumors as part of a phase III study. J Neurooncol 81:201-208

8. Glantz MJ, LaFollette S, Jaeckle KA et al (1999) Randomized trial of a slow-release versus a standard formulation of cytarabine for the intrathecal treatment of lymphomatous meningitis. J Clin Oncol 17:3110-3116

9. Glantz MJ, Jaeckle KA, Chamberlain MC et al (1999) A randomized controlled trial comparing intrathecal sustained release cytarabine (DepoCyt) to intrathecal methotrexate in patients with neo- plastic meningitis from solid tumors. Clin Cancer Res 5:3394-3402

10. Bomgaars L, Geyer JR, Franklin J et al (2004) Phase I trial of intrathecal liposomal cytarabine in children with neoplastic meningitis. J Clin Oncol 22:3916-3921

11. Jabbour E, O'Brien S, Kantarjian H et al (2007) Neurologic complications associated with intrathecal liposomal cytarabine given prophylactically in combination with high-dose methotrexate and cytarabine to patients with acute lymphocytic leukemia. Blood 109:3214-3218

12. Gökbuget N, Hartog CM, Bassan R et al (2011) Liposomal cytarabine is effective and tolerable in the treatment of central nervous system relapse of acute lymphoblastic leukemia and very aggressive lymphoma. Hematologica 96:238-244

13. Navajas A, Lassaletta A, Morales A et al (2012) Efficacy and safety of liposomal cytarabine in children with primary CNS tumours with leptomeningeal involvement. Clin Transl Oncol 14:280 286 